\title{
The Surface and Lower Atmosphere of Titan from HST Observations
}

\author{
Peter H. Smith, M. Lemmon, M. Tomasko \\ University of Arizona, Lunar \& Planetary Laboratory, Tucson, AZ \\ 85721, USA
}

\begin{abstract}
In 1994 the HST camera acquired images of Titan during 14 visits throughout a 16-day rotation period. These images were mosaicked to produce surface maps of albedo variations at several wavelengths $(0.67$, 0.94 , and 1.04 microns) which revealed a bright, continent-sized feature on Titan's leading face (Smith, Lemmon, \& Lorenz 1996). Also, the observing program was designed as a search for clouds and their motions; no clouds were conclusively identified. Other observing sessions in subsequent years performed by our group and others have resulted in numerous observations through WFPC2, STIS, and NICMOS. Additional maps have now been made in the 2 -micron region that show a very similar surface albedo to the 0.94 -micron map ( $\mathrm{T}$. Owen, private communication). Models based on these data sets are used to understand the lower atmosphere and surface as seen through the overlying hazes; seasonal variations resulting in a north-south asymmetry of the haze layer are also being studied.
\end{abstract}

\section{References}

Smith, P. H., Lemmon, M. T., \& Lorenz, R. D. 1996, Icarus, 119, 336 